\title{
EL NACIMIENTO DE LA NOVELA CORTA EN ESPAÑA (LA PERSPECTIVA DE LOS EDITORES)
}

\author{
JOSÉ MONTERO REGUERA \\ Universidad de Vigo
}

\begin{abstract}
F 1 radical cambio de opinión expresado por Lope de Vega en dos fechas separadas por casi veinte años muestra mejor que cualquier otro testimonio la evolución del género de la novela corta en España, desde un rechazo casi sin paliativos, hasta no sólo su aceptación, sino su práctica efectiva. Si en 1602 el autor de El peregrino en su patria, siguiendo una opinión muy común, censuraba este tipo de literatura así:
\end{abstract}

$\mathrm{Ni}$ es bien escrevir por términos tan inauditos que a nadie pareciessen inteligibles; pues si acaso las cosas son escuras, los que no han estudiado maldizen el libro, porque quisieran que todo estuviera lleno de cuentos y novelas: cosa indigna de hombres de letras; pues no es justo que sus libros anden entre mecánicos e ignorantes, que cuando no es para enseñar, no se ha de escrivir para los que no pudieron aprender; ${ }^{1}$

\footnotetext{
${ }_{1}$ Dedicatoria a don Juan de Arguijo en las Rimas (Sevilla, 1602/1604). Cito por Lope de Vega, Rimas, ed. crítica y anotada de Felipe B. Pedraza Jiménez, Fuenlabrada (Madrid), Servicio de Publicaciones de la Universidad de Castilla La Mancha, 1993, vol. I, p. 139. Para la opinión común de la época sobre la novela corta remito a los estudios clásicos de Agustín González de Amezúa: Formación y elementos de la novela cortesana, Madrid, Tipografía de Archivos, 1929, pp. 78-81, y Cervantes creador de la novela corta en española, Madrid, C.S.I.C., 1956, vol. I, pp. 244-260.
} 
por el contrario, en 1621, Lope no sólo incluye una novela corta en La Filomena, sino que, en competencia con Cervantes, afirma lo siguiente:

[...] en España [...] también hay libros de novelas, de ellas traducidas de italianos y de ellas propias, en que no le faltó gracia y estilo a Miguel de Cervantes. Confieso que son libros de grande entretenimiento y que podrían ser ejemplares, como algunas de las Historias trágicas del Bandelo, pero habían de escribirlos hombres científicos o por lo menos grandes cortesanos, gente que halla en los desengaños notables sentencias y aforismos. $^{2}$

En esos casi veinte años que van de 1602 a 1621 se produce un cambio singular en el parecer sobre la novela corta: serán las primeras partes del Guzmán de Alfarache (1599) y del Quijote (1605), y las cervantinas Novelas ejemplares (1613) las que influirán de manera decisiva en ese cambio de actitud. ${ }^{3}$

En efecto, la crítica suele atribuir a Miguel Cervantes (Novelas ejemplares) el paso definitivo para la implantación, adaptación y difusión de este género surgido en Italia en el siglo XIV, y conocido en España durante los siglos XV y XVI a través de traducciones de los textos italianos. ${ }^{4}$ Pero es realmente en la década de 1620-1630 cuando este género alcanza verdadero éxito y se suceden los títulos convirtiéndose a partir de entonces en la modalidad de ficción en prosa por excelencia del siglo XVII. La cronología, en este sentido, es muy reveladora, pues, según queda de manifiesto en el apéndice que incorporo al final de este trabajo, aunque hay algún otro ejemplo de novela corta en el período que va de 1600 a 1613, entre esta última fecha y 1620 apenas hay nada, salvo reediciones de los textos cervantinos (Quijote y Ejemplares) y de Alemán (Guzmán), pero no colecciones originales nuevas del novelas cortas. ${ }^{5}$ Estas aparecen en la década siguiente, cuando otros escritores (entre ellos algunos dramaturgos: Lope, Tirso, Pérez de Montalbán) lanzan al mercado un número importante de novelas cortas, generalmente (valga la excepción lopesca de las Novelas a Marcia Leonarda, 1621-1624), en forma de colección.

\footnotetext{
2 Lope de Vega, Novelas a Marcia Leonarda, edición, prólogo y notas de Francisco Rico, Madrid, Alianza Editorial, 1968, p. 28.

3 Remito al artículo de Maxime Chevalier, «La emergencia de la novela breve», Homenaje al profesor Antonio Vilanova, Barcelona, Universidad de Barcelona, 1989, vol. I, pp. 157-169.

4 Véase el volumen colectivo coordinado por José Luis Alonso Hernández, Martin Gosman y Rinaldo Rinaldi, La Nouvelle Romane (Italia - France - Espagne), Amsterdam y Atlanta (GA), Rodopi, 1993. Sigue siendo útil a este propósito el trabajo ya citado de González de Amezúa (Formación y elementos de la novela cortesana), que debe complementarse con el catálogo de Begoña Ripoll (La novela barroca, Salamanca, Universidad de Salamanca, 1991) y el libro de Anne Cayuela (Le paratexte au Siècle d'Or. Prose romanesque, livres et lecteurs en Espagne au XVIIe. siècle, Genève, Librairie Droz S. A., 1996).

${ }^{5}$ Sólo noto una excepción: los Días de jardín (1619), de Alonso Cano y Urrueta.
} 
Esas colecciones repiten además títulos parecidos o muy semejantes, que acaban corvirtiéndose en una suerte de reclamo publicitario que los escritores y editores saben aprovechar muy bien: un título breve que, generalmente, atiende a dos coordenadas: una espacial (jardín, cirgarral, Madrid, Toledo, Valencia, academia, casas, etc.) y otra temporal (noches, días, horas, etc.); en ocasiones se añaden variantes que suelen incidir en el aspecto moral y de entretenimiento (honesto, escarmiento, agradable, gustoso, apacible) y genérico (novela): Días de jardin, Tardes entretenidas, Jornadas alegres, Tiempo de regocijo y carnestolendas en Madrid, Noches de placer, Fiestas del jardin, Huerta de Valencia, Noches de invierno, Novelas ejemplares y prodigiosas, Academias del jardín, Las clavellinas de recreación, Cigarrales de Toledo, Deleitar aprovechando, etc. ${ }^{6}$

Si con el Guzmán y el primer Quijote, por un lado, y, por otro, el teatro, la literatura se había convertido -con la expresión cervantina- en "mercadería vendible» $(D Q, \mathrm{I}, 48)$, los autores $\mathrm{y}$, aún más, los editores van a aprovechar cuantos elementos proporcionen las obras literarias para obtener un beneficio económico. Así sucede también con la novela corta, como intentaré mostrar de un ejemplo poco conocido y no examinado -en lo que se me alcanza- desde esta perspectiva.

En efecto, la hoy muy olvidada figura del escritor portugués Manuel Faria y Sousa (1590-1649) ofrece perfiles interesantes que descubren a un escritor de una obra vasta, enciclopédica, que abarca los géneros más diversos, entre los que se hallarán los eruditos comentarios de Camoens (Os lusíadas [Madrid, 1639], Rimas varias de Camôes [Lisboa, 1685-1689]), los textos de carácter histórico (Epitome de las historias portuguesas [1628]) y poético (Divinas y humanas flores, primera y segunda parte [...] [Madrid, Diego Flamenco, 1624]). Entre esas obras destaco ahora Noches claras (Madrid, por la viuda de Cosme Delgado, 1623, según indica la página final, pero 1624 según la portada), que es elogiada así por Lope de Vega:

\author{
Peregrina erudición \\ de varias flores vestida, \\ enseñanza entretenida \\ y sabrosa corrección; \\ fuerzas del ingenio son, \\ dulce pluma y docta mano \\ de un filósofo cristiano, \\ Sosa, de las letras sol,
}

6 Aunque con otros propósitos, puede consultarse el capítulo dedicado a estas cuestiones por Anne Cayuela, ob. cit., pp. 248-265. 
José Montero Reguera

Demóstenes español,

y Séneca lusitano.

(Preliminares, 4v.)

El libro se divide en palestras («conversaciones de amigos, junta de discretos o corona de hombres», p. 6), la primera de las cuales sirve para explicar el marco: «Pues en estas nocturnas conversaciones, los circunstantes, oyentes doctos, se han pagado (justamente) de la variedad para la hermosura dellas [...] De suerte, que este nuestro ejercicio [=palestra], y este ha de ser el lugar dél en estas dilatadas noches del brumoso enero [...] Las noches serán alternadas, mas pues ha de ser nocturna la competencia, veamos si el tiempo de la noche nos conviene, y si es lícito que se interrumpa el imperio del sueño». (pp. 1, 11 y 11-12).

La obra se articula en siete noches, divididas a su vez en cinco palestras cada una. En cada palestra se trata un asunto distinto con abundante erudición. Se añaden al final una Tabla de las cosas notables (p. 502 y ss.) y un índice por noches De las materias principales deste libro. Es un volumen pretendidamente culto y erudito, una miscelánea en la que se tratan asuntos muy diversos que el propio autor califica en los siguientes términos, con una llamativa referencia a los libros de «patrañas» (i.e.: novelas):

(...) porque los bien intencionados no lo harán [hablar mal de su libro], y los otros tienen licencia para hacerlo, porque usan de su instituto [...] y más esta edad, que llegando un autor a un librero, le pregunta luego si el libro que trae es de patrañas, porque no se gasta aora otra cosa. Pero aquí (depuesta toda presunción) ofrezco libro, que el sabio puede llevar para su estudio, y la dama para su estrado: el que no fuere estudioso para su entretenimiento, y el que fuere dañado, para su embidia, o para su rabia, si rabia y embidia no es lo mismo.

(Prólogo, p. 11).

Hay una intención evidente por querer distinguir su obra del tipo de libros que se «gastaban» entonces, que no son otros sino las colecciones de novelas cortas. El dato es muy revelador, pues indica, por un lado, que a las alturas de 1623 los libros que tienen éxito y se venden son los de novelas cortas, pero, por otro, que el suyo no lo es; ¿por qué esa insistencia? ¿Hay algo en el libro que lo pudiera confundir con una colección de novelas cortas? Creo que sí, de ahí la explicación: el título, que recuerda a los de tantas colecciones que por esas fechas acaparaban los escaparates de las librerías madrileñas (y no sólo madrileñas). Noches claras podría remitir a las Noches áticas de Aulo Gelio -modelo sin duda de Faria-, pero al lector de entonces le evocaría antes títulos 
como los enumerados más arriba, que no la miscelánea del escritor latino del siglo segundo antes de Cristo. Y lo que ofrecía Faría era otra cosa. ${ }^{7}$

La explicación detallada de todo ello se hallará en otra obra conservada manuscrita en la Biblioteca Pública de Braga: Fortuna de Manuel de Faria y Sousa caballero de la orden de nuestro señor Jesucristo y de la Casa Real. ${ }^{8}$ Gracias a este texto conocemos sus avatares al servicio de nobles como el marqués de CastelRodrigo, sus viajes a Italia y a Madrid, ciudad que describe gráfica y satíricamente; sus pretensiones casi nunca satisfechas, sus relaciones con el conde-duque de Olivares, y, lo que me interesa más ahora, sus tiranteces con los editores de sus libros...

En el capítulo tercero del libro segundo Faria y Sousa relata los avatares de la publicación de sus Noches claras ${ }^{9}$, e indica que su título inicial era Discursos morales, políticos y satíricos, ${ }^{10}$ pero que el librero encargado de la impresión lo cambió por el que figuró finalmente en la portada:

\footnotetext{
El librero que se encargó de esta impresión presumía de estudiante y sabía bien cuánto las vanidades atraen a sí a los humanos o a los que tienen cuando menos tanto de bestias como de hombres, como si fueran progenie de sátiros y centauros; aunque yo creí que esto en él era menos ciencia que naturaleza. Díjome que la venta de un libro constaba de extravagancias en el decir y de algún título campanudo. Cuando yo le oí decir esto de campanudo, que fue voz nueva para mí, en títulos o frentes de libros, creí que en la frente de este mío había de ir pintada alguna campana. Informéme bien de lo que quería decir en esto, y díjome que no era lo que yo pensaba, sino que aunque las materias de mi libro estaban bien tratadas, el estilo era muy llano y el título nada peregrino, antes usado mucho, porque sobraban libros con el de moralidades y políticas, que no le imprimiría si
}

\footnotetext{
7 El propio Faria relata en su autobiografía (citada más abajo), que «Hallándome a la puerta del librero que le vendía [Noches claras], llegó un hombre a pedir otro libro que se intitula Dias de jardín, y estábale hojeando cuando llegó otro hombre, y reconociendo el libro dijo: Es muy bueno. Dos autores hubo ahora que, ambos a un tiempo, nos dio el uno muy buenos días y el otro muy buenas noches. Callé yo, aunque entendí que con galantería mucha había alabado mis noches y aquellos días; a ellos, por cierto, con más razón que a ellas». (The «Fortuna» [...], ed. Glaser, p. 92, nota). El libro al que se refiere el texto es el de Alonso Cano y Urreta, Días de Jardín, Madrid, Bernardino de Guzmán, 1619.

8 Véase ahora The «Fortuna» of Manuel de Faria e Sousa. An Autobiography. Introduction, Edition, Notes and Index By Edward Glaser, Münster Westfalen, Aschendorffsche Verlagsbuchhandlung, 1975.

${ }_{9}$ Ibídem, pp. 171-173.

10 Nótese la similitud del título con este otro de Juan Cortés de Tolosa, publicado en 1620: Discursos morales, Zaragoza, Juan de Naja, 1620. Ambos libros muestran la asimilación de la novela corta al mundo de las misceláneas, ya presente en el texto de Antonio de Eslava (Noches de invierno, 1609) y reiterado en obras posteriores, como la de Joseph Zatrilla y Vico, de título tan barroco: Engaños y desengaños del profano amor. Deducidos de la amorosa historia que a este intento se describe del duque don Federico de Toledo. Donde se reprehende lo dañoso de esta pasión, y se advierte su reparo en varios documentos morales y políticos. Exornados de toda erudición sacra y humana para mayor aprovechamiento de las almas, Nápoles, Jospeh Roseli, 1637.
} 
no le dejase poner otro título, y también ilustrar con una entrada ruidosa, del estilo moderno, y allá por dentro enjerir algunas cláusulas de esta estofa y que todo esto se incluía en la voz campanudo.

Cuando le oí estas cosas pensé morirme, primero de espanto y después de risa. Quísele persuadir a que excusásemos toda alteración, y echarle razones fue echar cruces en Berbería; como yo sabía el refrán de que dádivas quebrantan peñas, quise comprarle a dinero la porfía y ofrecíle doscientos reales; y ellos no obraron más que las razones; véase cómo obrarían ellas adonde ellos no obraban. Volviendo, con todo, a ellas, rendido ya en lo de la mudanza del título, le decía que, cuanto a lo del estilo, antes había menester [ser] moderado que añadido, al modo llamado brillante, porque [a] este contagio me había atraído la práctica de Madrid, y que teniendo estos discursos en el lenguaje que había escritos los suyos el célebre fray Héctor Pinto, los había salpicado de los modernos adornos. Esto era verdad. Al fin, no quiso rendirse y hube de rendirme yo porque me obligaba a ello la necesidad.[...]

Viéndome, pues, con esta necesidad y con el deseo de no abrir la puerta a la detención en Madrid, y creyendo que ganaría con la dedicatoria de este libro la buena gracia del secretario Francisco de Lucena, poderoso a cosas más difíciles; por no dilatarlo, consentí en lo que el librero quería, dándole todos mis poderes, no en derecho concedidos -como dicen los poderes públicos-, para que hiciese en la armonía de mis discursos más mudanzas de las que hacía cualquier músico o danzante, poniéndole solamente por condición inviolable que había de ser aprisa, pues ya le había manifestado la causa.

Cumplió él puntualmente con esto porque en menos de una semana me apareció con mi original, en que había varias transformaciones a espacios iguales: la primer[a] plana, tan otra que no tenía palabra que yo hubiese escrito; el título, que antes era como he dicho, venía a ser ahora el de Noches claras. Al verle y cotejarle con el estilo de las mudanzas y añadiduras, y principalmente con la primera plana, le dije estas formales palabras: Bien veo que vuestra merced, por ver que estos mis discursos se suponen en pláticas de algunos estudiosos que se juntaban en ciertas noches, al modo de las Áticas de Aulo Gelio, gustó de llamarlos Noches claras. El 'claras', aunque había luces en el aposento de estas conversaciones, extraño más que el 'Noches' por dos causas: una, porque pensarán que yo con este nombre me introduzco en el libro o en la sarta de claros varones, como conde Claros; otra, porque lo que viene añadido a estas Noches no las hace tan claras como oscuras, y así no corresponde el título a la obra. Diciéndoseme después de impreso el libro, que algunos de los que le leían, le acusaban con esta misma sentencia, respondía yo: Yo fui el primero que lo dije; y así, conténtense esos señores con la muestra que dan de cuerdos, que cuanto al dicho, mío es.

Tratamos luego de sacar las licencias y en el consejo real se encomendó el despacho del libro al oidor Gregorio López Madera, ministro grave y letrado y de gran cordura, aunque a mí, entonces, no me constaba tanto de esta verdad porque nunca le había tratado; mas sobró el tratarle ahora sólo en esto para constarme de ella enteramente, y fue de este modo. Yo, con el librero, fui a cobrar de la mano de este varón de seso el libro. Dándomelo me dijo esto: Yo no había menester ver la aprobación de este libro para despacharle, porque le he leído, y en lo que toca a la erudición y acierto con que están tratados los asuntos que contiene le tengo por digno de toda estimación. Pero de tiempos a tiempos, tiene unos modos de decir a lo moderno que no sólo desdicen de todo el otro estilo que lleva, mas le deslucen y hacen menos estimable; y el principio es de malísima digestión. Soy de parecer que vuestra merced le quite estas cosas por lo dicho, no porque ellas sean de calidad que obliguen a negarse la licencia de impresión.

Cuando yo oí esto a aquel ministro, que era lo que justamente podía salir por la boca del mismo Apolo, casi me eché por tierra besándole la mano por aquel cuerdo juicio, y brevemente le informé de lo que pasaba, con que él hizo más caso de mí, y después me 
El nacimiento de la novela corta en España (La perspectiva de los editores)

\begin{abstract}
trataba, cuando le veía, con particular respeto. Bajando de hablarle, detuve luego en el zaguán al librero y díjele: ¿Ha vuestra merced oído al señor Gregorio López Madera? ¿quiere más? Pues aunque hubiésemos acertado en esto, lo debíamos quitar en reverencia de haber parecido así a una tal persona. Respondióme: El señor Gregorio López no ha de vender este libro sino yo, y yo sé lo que me conviene para venderle. Casi estuvimos desacordados, mas soseguéme por afirmarme que en algo le templaría; aunque después vi que lo había hecho en bien poco. Hizo estampar en el rostro un sol, y díjome que con él nadie diría que las Noches no eran claras. Véase bien la claridad del juicio con quien yo trataba, para haberle de reducir con razones.

Finalmente dimos fin a la impresión del libro [...]
\end{abstract}

El texto es largo, pero muy interesante a nuestro propósito; de nada valieron los argumentos de Manuel Faria y Sousa, pues el librero hizo prevalecer siempre su punto de vista de mercader de libros, de vendedor de un producto: «y yo sé lo que me conviene para venderle», sentencia rotundo. Y para ello necesita un título «campanudo», asociado en este caso a los que tanta fama iban teniendo en las colecciones de novela corta; ciertas concesiones al «estilo moderno», al «modo brillante» que triunfa en la época, esto es, un cierto tono gongorizante (el estilo del escritor era «muy llano»); y una portada atractiva, a través de un grabado que llamara la atención. No importaba que el título no se acomodara bien al contenido, se trataba de ofrecer en los inicios del mismo una serie de elementos de moda que atrajeran no tanto a un posible lector, sino a un potencial comprador. Sin duda hay toda una estrategia editorial tras las palabras del editor de Noches claras. ${ }^{11}$

Gracias a la imprenta, la literatura se ha convertido en un auténtico negocio que podía ofrecer pingües beneficios; el Guzmán de Alfarache mostró a a las claras cómo los editores podían ganar dinero (y mucho) con ella. ${ }^{12}$

\footnotetext{
11 Acaso fuera Andrés de Carrasquilla, a cuya costa se había publicado en 1620 la Casa del placer honesto, de Salas Barbadillo. Por las mismas fechas de las Noches claras, entre 1623 y 1624, en la imprenta de la viuda de Cosme Delgado sale a la luz El curial del Parnaso, de Matías de los Reyes, con textos preliminares de Lope de Vega y Juan Pablo Martir Rizo. Curiosamente, si el texto de Faria incluye al principio (noche primera, palestra V) un capítulo sobre «Qué es amistad. A los amigos que agora se usan, que no sirven de nada», Matías de los Reyes incluye un Aviso II sobre «En que se muestra cómo han de ser los amigos. Pone por ejemplo un caso de dos amigos en que se verifica esta proposición» (ed. Madrid, Librería de los Bibliófilos Españoles, 1909, p. 30 y ss.). No acaban ahí las similitudes entre Noches claras y El curial del Parnaso.

12 Véanse a este respecto, Francisco Márquez Villanueva, «Sobre el lanzamiento y recepción del Guzmán de Alfarache», Bulletin Hispanique, 92, 1 (1990), pp. 549-577; José María Micó, «Prosas y prisas en 1604: El Quijote, el Guzmán y la Pícara Justina», Hommage à Robert Jammes, Toulouse, P.U.M., 1994, pp. 827-448; y, especialmente, Francisco Rico, Visita de imprentas. Páginas y noticias de Cervantes Viejo. Discurso pronunciado por Franciso Rico el 10 de mayo de 1996 en ocasión de su investidura como doctor honoris causa por la Universidad de Valladolid [...], s.1., 1996, revisado y aumentado en «A pie de imprentas. Páginas y noticias de Cervantes viejo», Bulletin Hispanique, 2 (2002), pp. 673-702.
} 
Recientemente, Juan Carlos Rodríguez ha querido ver en el Quijote, «un texto directamente comercial», «el primer libro laico que expresa directamente y sin tapujos su intención: está escrito para ser leído en masa [...], es decir, en busca de cualquier tipo de público»; y en su autor a un escritor profesional que «asume su último oficio completamente en serio» ${ }^{13}$, aunque quizás todo esto se muestre, creo, más patente en otros libros cervantinos. ${ }^{14}$ En todo caso, con matizaciones, es un proceso que se inicia en torno a 1600 y que se muestra más que asentado y conocido en torno a 1620. El texto autobiográfico de Faria y Sousa lo ejemplifica muy bien, a la par que hace patente que el género de la novela corta nace, en el interés de los editores (y libreros), en torno a esa misma fecha.

13 Juan Carlos Rodríguez, El escritor que compró su propio libro. Para leer el "Quijote», Barcelona, Debate, 2003, pp. 59, 9 y 60 respectivamente.

14 Véanse mis trabajos, «Los preliminares del Persiles: estrategia editorial y literatura de senectud», Jean Pierre Sánchez, coord., Lectures d'une oeuvre: Los trabajos de Persiles y Sigismunda, Nantes, Éditions du Temps, 2003, pp. 65-78, y «Dos libros «'Para leer el Quijote'», Revista de Libros, 85 (enero 2004), pp. 39-40. 


\section{Apéndice}

\section{Listado cronólogico de colecciones de novelas cortas publicadas en el primer tercio del siglo XVII ${ }^{15}$}

1602-1603: Diego de Ávalos y Figueroa, Primera parte de la miscelánea austral de D. [...], en varios coloquios, interlocutores, Delio y Cilena, con la defensa de las damas, Lima, Antonio Ricardo, 1602 y 1603.

1604: Juan de Arce Solórzano, Tragedias de amor, de gustoso y apacible entretenimiento de historias, fábulas, enredadas marañas, cantares, bayles, ingeniosas moralidades de enamorado, Madrid, Juan de la Cuesta, 1604.

1609: Antonio de Eslava, Noches de invierno, Pamplona, Carlos de Labayen, 1609.

1613: Miguel de Cervantes Saavedra, Novelas ejemplares, Madrid, Juan de la Cuesta, 1613; Diego Rosel y Fuenllana, Parte primera de varias aplicaçiones, y transformaciones, las quales tractan, terminos cortesanos, práctica militar, casos de estado, en prosa y verso con nueuos hieroglíficos, y algunos puntos morales, Nápoles, Juan Domingo Roncallolo, 1613.

1614: Ambrosio de Salazar, Las clavellinas de recreación. Donde se contienen sentencias, avisos, exemplos y historias muy agradables para todo género de personas deseosas de leer cosas curiosas [...], Rouen, Adrien Morront, 1614. 1617.

1617: Juan Cortés de Tolosa, Discursos morales, Zaragoza, Juan de Naja,

1619: Alonso Cano y Urrueta, Días de jardín, Madrid, Bernardino de Guzmán, 1619.

1620: Juan Cortés de Tolosa, Lazarillo de Manzanares con otras cinco novelas, Madrid, viuda de Alonso Martín, 1620; Alonso Gerónimo de Salas Barbadillo, Casa del placer honesto, Madrid, viuda de Cosme Delgado, 1620; Antonio Liñán y Verdugo, Guía y aviso de forasteros, a donde se les enseña a huir de los peligros que hay en la vida de corte y debajo de novelas morales y ejemplares escarmientos [...], Madrid, viuda de Alonso Martín, 1620.

1621: Lope de Vega, Las fortunas de Diana, incluida en La Filomena, Madrid, viuda de Alonso Martín, a costa de Alonso Pérez, 1621, ff. 59-75v.

1622: Francisco Lugo Dávila, Teatro popular. Novelas morales [...], Madrid, Alonso Pérez, 1622.

${ }^{15}$ La lista que ofrezco es una selección en la que no incluyo reediciones de textos, ni incorporo obras extensas con novelas cortas interpoladas. Sí incluyo, en cambio, otros títulos que, aun no correspondiendo estrictamente con el género, muestran algunos elementos de semejanza. Me he basado fundamentalmente en el catálogo elaborado por Anne Cayuela, ob. cit., pp. 332-383. 
José Montero Reguera

1622 ó 1623/1636: Matías de los Reyes, El Menandro, Jaén, Francisco Pérez de Castilla, 1636. Según indica Emilo Cotarelo (ed. de Matías de los Reyes, El Menandro, Madrid, Librería de los Bibliófilos Españoles, 1909, p. XXIX), el libro debió de escribirse en 1622 ó 1623, en todo caso antes de 1624, pues la aprobación firmada por el «Doctor Francisco Ortiz de Peñafiel», lleva fecha de 20 de abril de 1624 (ed. cit., p. XXVIII)

1623/1624: Manuel Faria y Sousa, Noches claras, Madrid, viuda de Cosme Delgado, 1623, indica la página final, pero 1624 según la dedicatoria a Francisco de Lucena, 7 de febrero de 1624, y portada.

1624: Juan Pérez de Montalbán, Sucesos y prodigios de amor en ocho novelas ejemplares, Madrid, Juan González, 1624; Juan de Piña, Novelas ejemplares y prodigiosas, Madrid, Juan González, 1624; Matías de los Reyes, El curial del Parnaso, Madrid, viuda de Cosme Delgado, 1624; Tirso de Molina, Cigarrales de Toledo, Madrid, Luis Sánchez, 1624; Lope de Vega, La desdicha por la honra, La prudente venganza y Guzmán el bravo, incluidas en La Circe, con otras rimas y prosas, Madrid, viuda de Alonso Martín, a costa de Alonso Pérez, 1624, ff. 109$149 \mathrm{v}$.

1625: Alonso de Castillo Solórzano, Tardes entretenidas, Madrid, viuda de Alonso Martín, 1625; Baltasar Mateo Velázquez, El filósofo de aldea, y sus conversaciones familiares y ejemplares por casos y sucesos casuales, Madrid, Diego Flamenco, 1625.

1626: Alonso de Castillo Solórzano, Jornadas alegres, Madrid, Juan González, 1626; Francisco de Quintana, Experiencias de amor y fortuna, Madrid, viuda de Alonso Martín, 1626.

1627: Alonso de Castillo Solórzano, Tiempo de regocijo y carnestolendas en Madrid, Madrid, Luis Sánchez, 1627; Salvador Jacinto Polo de Medina, Academias del jardin, Madrid, Juan González, 1627.

1628: Alonso de Castillo Solórzano, Escarmientos de amor moralizados, Sevilla, Manuel Sande, 1628; Juan de Piña, Casos prodigiosos y cueva encantada, Madrid, Imprenta del Reino, 1628; Miguel Moreno, El cuerdo amante, 1628.

1629: Alonso de Castillo Solórzano, Huerta de Valencia, Valencia, Miguel Sorolla, 1629; Juan de Piña, Segunda parte de los casos prodigiosos, Madrid, viuda de Alonso Martín, 1629.

1631: Alonso de Castillo Solórzano, Noches de placer, Barcelona, Sebastián de Cormellas, 1631.

1632: Juan Pérez de Montalbán, Para todos. Ejemplos morales, humanos y divinos. En que se tratan diversas ciencias, materias y facultades, repartidas en los siete días de la semana, Madrid, Imprenta del Reino, 1632.

1633: Alonso de Castillo Solórzano, Las harpías en Madrid y coche de las estafas, Barcelona, Sebastián de Cormellas, 1633. 
El nacimiento de la novela corta en España (La perspectiva de los editores)

1634: Alonso de Castillo Solórzano, Fiestas del jardín [...], Valencia, Silvestre Esparsa, 1634.

1635: Tirso de Molina, Deleitar aprovechando, Madrid, Imprenta Real, 1635.

LECTURA Y SIGNO, 1 (2006) pp. 165-175 